\title{
Erastin decreases radioresistance of NSCLC cells partially by inducing GPX4-mediated ferroptosis
}

\author{
XIAOFEN PAN ${ }^{1,2^{*}}$, ZHIXIU LIN $^{3 *}$, DANXIAN JIANG $^{1 *}$, YING YU ${ }^{1}$, DONGHONG YANG ${ }^{1}$, HECHAO ZHOU ${ }^{1}$, \\ DECHAO ZHAN ${ }^{1}$, SHA LIU ${ }^{4}$, GANG PENG ${ }^{5}$, ZIHONG CHEN $^{1}$ and ZHONGHUA YU $^{1}$ \\ ${ }^{1}$ Department of Head and Neck Cancer, Oncology Center, Affiliated Hospital of Guangdong Medical College, Zhanjiang, \\ Guangdong 524000; ${ }^{2}$ Department of Oncology, The Seventh Affiliated Hospital, Sun Yat-sen University, Shenzhen, \\ Guangdong 518000; ${ }^{3}$ Department of Pharmacy, Affiliated Hospital of Guangdong Medical College, Zhanjiang, \\ Guangdong 524000; ${ }^{4}$ Department of Oncology, Jingzhou Central Hospital, Jingzhou, Hubei 434020; \\ ${ }^{5}$ Department of Head and Neck Cancer, Cancer Center, Union Hospital, Tongji Medical College, \\ Huazhong University of Science and Technology, Wuhan, Hubei 430030, P.R. China
}

Received September 24, 2016; Accepted November 16, 2018

DOI: $10.3892 /$ ol.2019.9888

\begin{abstract}
The aim of the present study was to examine whether erastin influences radioresistance in non-small cell lung cancer (NSCLC) cells and produce a preliminary investigation into its mechanism of action. The radioresistant subtype of NSCLC cells, A549-R and H460-R, were induced by high-dose hypofractionated irradiation. Erastin was used to treat the radioresistant cells and radiosensitivity was examined by colony formation assays. Cell death was determined after the cells were treated with erastin, irradiation (IR) or erastin together with IR. The expression of glutathione peroxidase 4 (GPX4) expression in the parental cells and radioresistance cells was detected by western blotting. GPX4 expression in the radioresistance cells was subsequently inhibited, radiosensitivity and cell death was measured, and erastin enhanced radiosensitivity in A549-R and H460-R cells. Erastin and IR exhibited a combined effect on killing cells, as co-treatment with erastin and IR demonstrated a higher effect on killing cells compared with erastin or IR alone. GPX4 expression was inhibited by erastin in the radioresistant cells. Inhibiting GPX4 expression also radiosensitized NSCLC cells to radiation in the radioresistant cell lines. Erastin-induced and GPX4-inhibition-induced cell death could partially be
\end{abstract}

Correspondence to: Dr Zhonghua Yu or Dr Zihong Chen, Department of Head and Neck Cancer, Oncology Center, Affiliated Hospital of Guangdong Medical College, 57 Southern of Renmin Road, Zhanjiang, Guangdong 524000, P.R. China

E-mail: zhonghua_yu@126.com

E-mail: chen.zi.hong@163.com

${ }^{*}$ Contributed equally

Key words: erastin, ferroptosis, glutathione peroxidase 4, radioresistance, non-small cell lung cancer rescued by deferoxamine, but not Z-VAD-FMK and olaparib, which indicated that erastin and GPX4-inhibition induced ferroptosis in the radioresistant cells. Erastin decreased radioresistance of NSCLC cells partially by inducing GPX4-mediated ferroptosis.

\section{Introduction}

Lung cancer is one of the most malignant types of cancer in humans, and according to the 2015 National Institutes of Health statistics, non-small cell lung cancer (NSCLC) constitutes $\sim 83.9 \%$ of lung cancer cases in the USA $(1,2)$. Radiotherapy is one of the principal therapeutic strategies in the treatment of lung cancer (3). However, resistance to radiation results in a poor outcome following radiotherapy $(4,5)$. Thus, identifying novel methods to avoid radiation resistance and improve the efficacy of radiotherapy is a major challenge in the treatment of lung cancer.

Erastin was originally identified when Dolma et al (6) screened small molecules that are synthetically lethal in cancer cells expressing RAS (6,7). Erastin induces iron-dependent cell death that is morphologically, biochemically and genetically different from apoptosis, necrosis, necroptosis and autophagy, and this form of cell death is termed ferroptosis (8-11). Recently, Yu et al (12) reported that erastin enhances sensitivity of AML cells to chemotherapy. Another study reported that the inhibition of ferroptosis increases sorafenib resistance in hepatocellular carcinoma cells (13). Ivanov et al $(14,15)$ described that iron-containing water improves the efficiency of radiotherapy and deferoxamine (DFOM) reduces the efficiency of radiotherapy in animals with gliomas. These data indicated that ferroptosis participates in the regulation of response to chemotherapy and radiotherapy in cancer cells.

According to the Lung Cancer Mutation Consortium database, oncogenic K-ras mutations were identified in $23 \%$ of patients with lung cancer between 2009-2015 (16). Erastin is a RAS-selective lethal (RSL) compound, which can trigger ferroptosis (17). Herein, we hypothesized that erastin may 
influence radiosensitivity in NSCLC by triggering ferroptosis. In the present study, two K-ras-expressing NSCLC cell lines (A549 and H460) were selected to conduct the experiments $(18,19)$. The present study aimed to verify that erastin can decrease, at least partially, the radioresistance of NSCLC cells and attempted to perform a preliminary investigation regarding the molecular mechanism.

\section{Materials and methods}

Reagents. The RPMI-1640 culture medium was purchased from Gibco (Thermo Fisher Scientific, Inc., Waltham, MA, USA). Fetal bovine serum (FBS) was purchased from Hangzhou Sijiqing Biological Engineering Materials Co., Ltd (Hangzhou, China). Dimethyl sulfoxide (DMSO), DFOM, Z-VAD-FMK, trypsin and trypan blue were purchased from Sigma-Aldrich (Merck KGaA, Darmstadt, Germany). Primers for glutathione peroxidase 4 (GPX4), small interfering (si) RNA of GPX4, Lipofectamine ${ }^{\circledR} 2000$, TRIzol ${ }^{\circledR}$, OPTI-MEM I, MMLV reverse transcriptase, Taq DNA polymerase and Oligo dT primers were purchased from Invitrogen (Thermo Fisher Scientific, Inc.). Erastin and olaparib were purchased from Selleck Chemicals (Houston, TX, USA). Protein molecular weight standards were purchased from Fermentas (Thermo Fisher Scientific, Inc.). Protein lysis buffer and the Bicinchoninic Acid (BCA) protein assay kit were purchased from the Beyotime Institute of Biotechnology (Wuhan, China). Protease inhibitors were purchased from Roche Diagnostics (Basel, Switzerland). Rabbit anti-GPX4 (catalog no. ab125066; $1: 1,000)$ and anti- $\beta$-actin (catalog no. ab8226; $1: 500$ ) were purchased from Abcam (Cambridge, MA, USA). Horseradish peroxidase (HRP)-conjugated goat anti-rabbit secondary antibody (catalog no. TA130023; 1:3,000) was purchased from OriGene Technologies, Inc. (Beijing, China). All antibodies were diluted in bovine serum albumin. The Enhanced Chemiluminescence (ECL) chemiluminescence reagent was purchased from Thermo Fisher Scientific, Inc.

Cell culture. The human NSCLC cell lines A549 and H460 were purchased from the American Type Culture Collection (Manassas, VA, USA). Cells were cultured in RPMI-1640 medium supplemented with 10\% FBS, 100 IU penicillin and $100 \mu \mathrm{g} / \mathrm{ml}$ streptomycin, and incubated at $37^{\circ} \mathrm{C}$ in a $5 \% \mathrm{CO}_{2}$ humidified incubator.

Establishment of the NSCLC radioresistant subtype cell lines. Exponentially growing NSCLC cells A549 and H460 were irradiated with 5 does of $6 \mathrm{~Gy}$. Irradiation was performed with 6-MV X-rays generated by a Siemens Primus H high-energy linear accelerator (Siemens Healthineers, Erlangen, Germany), as described previously $(20,21)$. There was a $7-9$ day break in between each irradiation (21). The radiation field was $10 \times 10 \mathrm{~cm}$, the distance from the source to target was $100 \mathrm{~cm}$ and the absorbed dose rate was $0.2 \mathrm{~Gy} / \mathrm{min}$. The surviving sublines (A549-R and H460-R) were then passaged for three months at $37^{\circ} \mathrm{C}$ and the radiosensitivity was determined by colony formation assays.

Colony formation assay. A single cell suspension was prepared from the A549, A549-R, H460 and H460-R cells in their logarithmic growth phase using $0.25 \%$ trypsin for digestion. Cells were seeded in 6-well plates and received single dose irradiation of $0,2,4,6$ and 8 Gy after a $12 \mathrm{~h}$ culture adherence at $37^{\circ} \mathrm{C}$ in a $5 \% \mathrm{CO}_{2}$ humidified incubator. Cell density was 200, 300, 600, 1,200 and 2,400 cells/well for each radiation dose. After 2 weeks, cells were fixed with $4 \%$ paraformaldehyde at $25^{\circ} \mathrm{C}$ for $20 \mathrm{~min}$ and were stained with Giemsa at $25^{\circ} \mathrm{C}$ for $15 \mathrm{~min}$. Cell survival fraction (SF) was calculated based on the following formula: $\mathrm{SF}=$ colony numbers in experimental group/[plating number $\mathrm{x}$ plating efficiency $(\mathrm{PE})] . \mathrm{PE}=($ colony numbers in the control group/plating number in control group) $\mathrm{x} 100 \%$. A cell survival curve was obtained by the single-hit multi-target model [SF=l- $\left.\left(1-\mathrm{e}^{-\mathrm{D} / \mathrm{D} / \mathrm{N}}\right)^{\mathrm{N}}\right]$ using Sigma Plot 10.0 version (Systat Software, Inc, CA, USA). The parameters of cell survival fraction, a 2 Gy irradiation dose (survival fraction at $2 \mathrm{~Gy}$ irradiation, $\mathrm{SF}_{2}$ ), mean lethal dose value $\left(\mathrm{D}_{0}\right)$, quasithreshold dose $\left(\mathrm{D}_{\mathrm{q}}\right)$ and extrapolation number $(\mathrm{N})$ were calculated. Higher values of $\mathrm{SF}_{2}, \mathrm{D}_{0}$ and $\mathrm{Dq}$ indicated an increased radioresistance (22).

Cell death assay. A549-R and H460-R cells were divided into four groups for the following treatments: i) control group treated with DMSO; ii) treated with $4 \mu \mathrm{M}$ erastin alone for $12 \mathrm{~h}$ at $37^{\circ} \mathrm{C}$; iii) treated with $2 \mathrm{~Gy}$ IR alone and cultured in medium without erastin for $12 \mathrm{~h}$ at $37^{\circ} \mathrm{C}$; and iv) treated with 2 Gy IR and then cultured in medium with $4 \mu \mathrm{M}$ erastin for $12 \mathrm{~h}$. Following the indicated treatments, the cells were trypsinized and stained with trypan blue for $3 \mathrm{~min}$ at $25^{\circ} \mathrm{C}$, followed by counting with a hemocytometer using a standard protocol (21). Cells stained blue were considered as dead cells.

In addition, A549-R and H460-R cells were divided into eight groups for the following treatments: i) control group; ii) treated with $100 \mu \mathrm{M}$ DFOM; iii) treated with $50 \mu \mathrm{M}$ Z-VAD-FMK; iv) treated with $10 \mu \mathrm{M}$ olaparib; v) treated with $4 \mu \mathrm{M}$ erastin; vi) treated with $4 \mu \mathrm{M}$ erastin and $100 \mu \mathrm{M}$ DFOM; vii) treated with $4 \mu \mathrm{M}$ erastin and $50 \mu \mathrm{M} \mathrm{Z-VAD-FMK}$; and viii) treated with $4 \mu \mathrm{M}$ erastin and $10 \mu \mathrm{M}$ olaparib. All groups were incubated with an equal volume DMSO, including the control group. The eight groups of cells were treated for $12 \mathrm{~h}$ at $37^{\circ} \mathrm{C}$. Following the indicated treatments, the cells were trypsinized and stained with trypan blue for $3 \mathrm{~min}$ at $25^{\circ} \mathrm{C}$, followed by counting with a hemocytometer using the standard protocol (23). Cells stained blue were considered as dead cells.

Western blotting. A549, A549-R, H460 and H460-R cell lysates were prepared using the protein lysis buffer, according to the manufacturer's protocol. Following extraction, protein concentration was determined with a BCA assay. A total of $30 \mu \mathrm{g}$ protein from each sample was loaded into a $10 \%$ SDS-PAGE, followed by a transfer to polyvinylidene fluoride membranes (EMD Millipore, Billerica, MA, USA). Membranes were blocked with 5\% bovine serum albumin (catalog no. ST023-1000 g; Beyotime Institute of Biotechnology) for $2 \mathrm{~h}$ at $37^{\circ} \mathrm{C}$. They were then incubated with rabbit anti-GPX4 $(1: 1,000)$ and anti- $\beta$-actin (1:500) antibodies at $4^{\circ} \mathrm{C}$ overnight. The polyvinylidene fluoride membrane was washed three times with TBS and Tween-20, 5 min each. Subsequently, the membranes were incubated with HRP-conjugated goat anti-rabbit $(1: 3,000)$ antibodies for $1 \mathrm{~h}$ at $25^{\circ} \mathrm{C}$. The ECL reagent then was added to the membranes and the optical density of the bands was 
analyzed using the Image J V1.8.0 software (National Institute of Health, Bethesda, MD, USA).

siRNA transfection. siGPX4 (catalog no. AM16708) and the control siRNA (siMock; catalog no. AM4611) were obtained from Invitrogen (Thermo Fisher Scientific, Inc.). A solution composed of $1 \mathrm{ml}$ OPTI-MEM, $6 \mu$ l Lipofectamine 2000 and $2 \mu \mathrm{l}$ siRNA (the final concentration was $2 \mu \mathrm{l} / \mathrm{ml}$ ) was prepared and the mixture $(1 \mathrm{ml} /$ well) was incubated in a 6 -well dish for $20 \mathrm{~min}$ at $37^{\circ} \mathrm{C}$. Following incubation, 200,000 cells were suspended in $1 \mathrm{ml}$ RPMI-1640 culture medium with $20 \%$ FBS (Thermo Fisher Scientific, Inc.). Subsequently, they were transferred to each well and incubated for $72 \mathrm{~h}$. GPX4 expression was measured by western blot analysis, as aforementioned.

Statistical analysis. Statistical analysis was performed using SPSS 17.0 (SPSS Inc., Chicago, IL, USA). Data are presented as the mean \pm standard error of the mean. Significant differences between the groups were determined by unpaired Student's t-test and by one-way analysis of variance followed by a Bonferroni post hoc test for multiple group comparisons (Figs. 3, 4 and 7). $\mathrm{P}<0.05$ was considered to indicate a statistically significant difference.

\section{Results}

Radiosensitivity change in A549-R and H460-R cells. To confirm that the radioresistant cell line was successfully established, a colony formation assay was performed to determine the radiosensitivity of A549-R and H460-R cells. The results demonstrated that A549-R and H460-R cells had increased survival fractions, compared with A549 and H460 cells, respectively. Cell survival curves were used to analyze the results. Therefore, the shoulder area under the survival curve was increased in H460-R and A549-R cells (Fig. 1). Compared with the A549 and H460 cells, A549-R and $\mathrm{H} 460-\mathrm{R}$ cells, respectively, had increased $\mathrm{D}_{0}, \mathrm{D}_{\mathrm{q}}, \mathrm{N}$ and SF2 values (Table I), which represented increased radioresistance. Collectively, these results demonstrated that radioresistant (A549-R and H460-R) cells were successfully established.

Erastin enhances radiosensitivity in A549-R and H460- $R$ cells. A colony formation assay was performed to investigate the effect of erastin on radiosensitivity of A549-R and H460-R cells. The results demonstrated that erastin-treated groups had reduced survival fractions, compared with the control groups. Cell survival curves were used to analyze the results. Additionally, the shoulder area under the survival curves was reduced in the erastin-treated groups, compared with the control groups (Fig. 2). Compared with the control group cells, the erastin group cells had decreased $\mathrm{D}_{0}, \mathrm{D}_{\mathrm{q}}, \mathrm{N}$ and $\mathrm{SF}_{2}$ values (Table II), which indicated decreased radioresistance. The result demonstrated that erastin enhances the sensitivity to radiation therapy in the radioresistant NSCLC cells.

Erastin and IR exhibit a combined effect on killing cells. Following indicated treatment, cell death was measured. In A549-R cells, cell death fraction induced by erastin + IR was $60.2 \pm 2.7 \%$. The cell death percentage induced by erastin
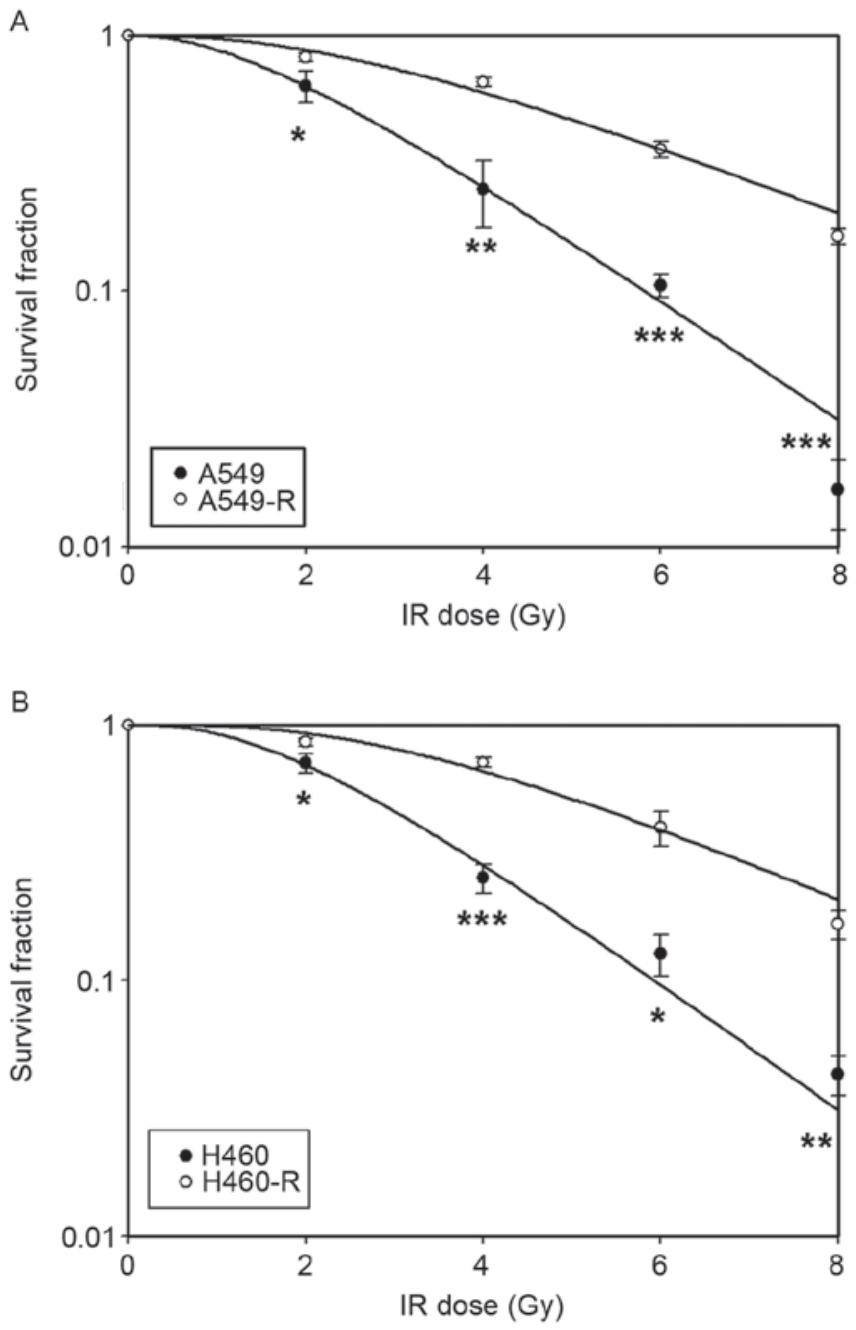

Figure 1. Clone formation assay determining the radiosensitivity of A549-R and H460-R cells. The results demonstrated that A549-R and H460-R cells had increased survival fractions, compared with A549 and H460 cells, respectively. The shoulder area under the survival curve was increased in H460-R and A549-R cells. (A) Radiosensitivity of A549 and A549-R cells. (B) Radiosensitivity of H460 and H460-R cells. Data are presented as the mean \pm standard deviation. ${ }^{*} \mathrm{P}<0.05,{ }^{* *} \mathrm{P}<0.01,{ }^{* * *} \mathrm{P}<0.001$ A549-R vs. A549 or H460-R vs. H460. R, radioresistant; IR, irradiation.

alone was $32.5 \pm 1.9 \%$, while the IR-induced cell death fraction was $19.1 \pm 2.5 \%$. In H460-R cells, the cell death fraction in the erastin + IR group was significantly increased, compared with the erastin or IR groups $(39.4 \pm 4.5 \%$ vs. $21.2 \pm 2.2 \%$ or $14.9 \pm 2.1 \%$, respectively) (Fig. 3 ). These data revealed that erastin and IR exhibited a combined effect on killing cells.

Erastin-induced cell death could partially be rescued by DFOM instead of Z-VAD-FMK and olaparib. DFOM is an iron chelator, which can inhibit ferroptosis (24). Z-VAD-FMK is a caspase-inhibitor, which can inhibit caspase-dependent apoptosis (25). Olaparib is a poly(ADP-ribose) polymerase 1 (PARP-1) inhibitor that can inhibit PARP-1-dependent apoptosis (26). Cell death was detected following treatment with DFOM $(100 \mu \mathrm{M})$, Z-VAD-FMK $(50 \mu \mathrm{M})$ and olaparib $(10 \mu \mathrm{M})$ alone or with erastin $(4 \mu \mathrm{M})$ for $12 \mathrm{~h}$ at $37^{\circ} \mathrm{C}$. Treatment with erastin induced notable cell death. Treating cells with DFOM, Z-VAD-FMK and olaparib alone had no notable effect on 
Table I. Parameters of radiosensitivity in different cell lines.

\begin{tabular}{lcccc}
\hline Parameter & A549 & A549-R & H460 & H460-R \\
\hline $\mathrm{D}_{0}$ & 1.84 & 3.12 & 1.74 & 2.81 \\
$\mathrm{D}_{\mathrm{q}}$ & 1.50 & 2.17 & 1.70 & 2.40 \\
$\mathrm{~N}$ & 2.43 & 2.80 & 3.14 & 3.92 \\
$\mathrm{SF}_{2}$ & 0.59 & 0.85 & 0.52 & 0.81 \\
\hline
\end{tabular}

$\mathrm{D}_{0}$, mean lethal dose; $\mathrm{D}_{\mathrm{q}}$, quasi-threshold dose; $\mathrm{N}$, extrapolation number; $\mathrm{SF}_{2}$, surviving fraction at $2 \mathrm{~Gy} ; \mathrm{R}$, radioresistant.
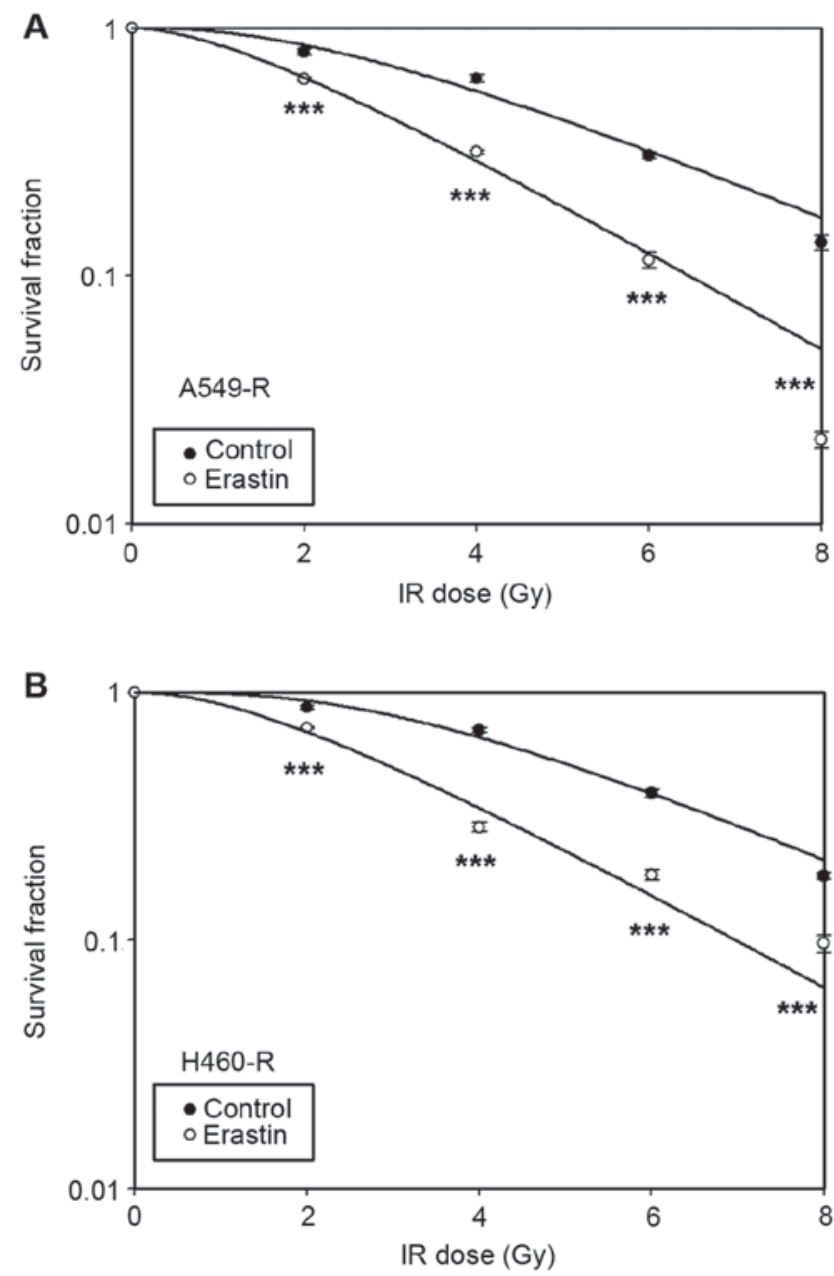

Figure 2. Colony formation assay investigating the effect of erastin on radiosensitivity of A549-R and H460-R. The results demonstrated that groups treated with erastin had reduced survival fractions, compared with the control groups. The shoulder area under the survival curves was reduced in erastin groups. (A) Radiosensitivity of A549-R cells. (B) Radiosensitivity of H460-R cells. Data are presented as the mean \pm standard deviation. ${ }^{* * *} \mathrm{P}<0.001$ vs. control. R, radioresistant; IR, irradiation.

cell death. When cells were treated with DFOM and erastin together, cell death was significantly decreased, compared with treated with erastin alone. However, when cells were treated with Z-VAD-FMK and erastin, cell death exhibited no significant difference, compared with treated with erastin alone. Additionally, olaparib had no significant effect on erastin-induced cell death (Fig. 4). The results indicated that
Table II. Parameters of radiosensitivity in different groups.

\begin{tabular}{llllll}
\hline & \multicolumn{2}{c}{ A549-R } & & \multicolumn{2}{c}{ H460-R } \\
\cline { 2 - 3 } \cline { 5 - 6 } Parameter & Control & Erastin & & Control & Erastin \\
\hline $\mathrm{D}_{0}$ & 2.97 & 2.21 & & 2.85 & 2.28 \\
$\mathrm{Dq}$ & 2.08 & 1.45 & & 2.39 & 1.61 \\
$\mathrm{~N}$ & 2.71 & 1.93 & & 3.82 & 2.20 \\
$\mathrm{SF}_{2}$ & 0.85 & 0.63 & & 0.93 & 0.69
\end{tabular}

$\mathrm{D}_{0}$, mean lethal dose; $\mathrm{D}_{\mathrm{q}}$, quasi-threshold dose; $\mathrm{N}$, extrapolation number; $\mathrm{SF}_{2}$, surviving fraction at $2 \mathrm{~Gy} ; \mathrm{R}$, radioresistant.

\section{A}

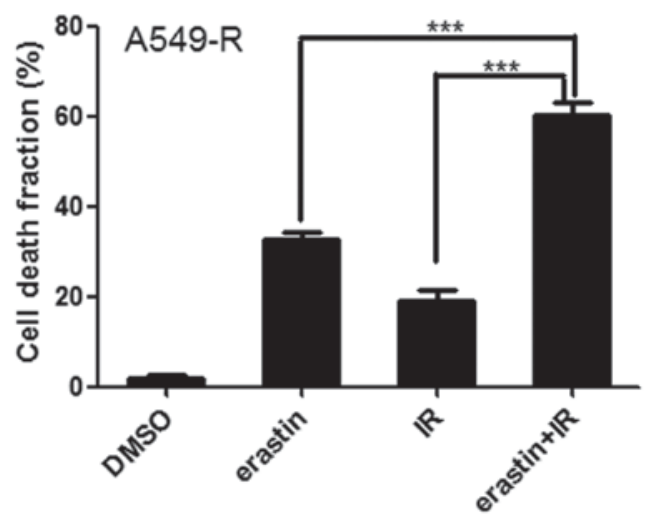

B

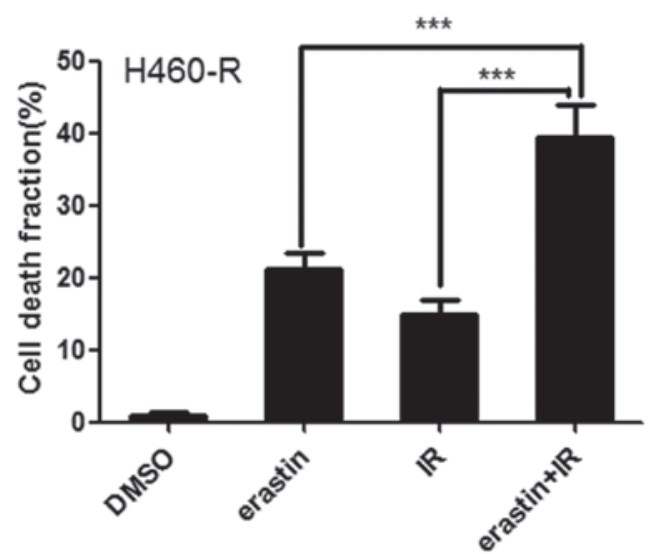

Figure 3. Cell death measurements following the administration of different treatments. The cell death fraction in the erastin + IR group was significantly increased, compared with the erastin or IR groups. (A) Cell death fraction in A549-R cells. (B) Cell death fraction in H460-R cells. Data are presented as the mean \pm standard deviation. ${ }^{* * *} \mathrm{P}<0.001$. R, radioresistant; IR, irradiation; DMSO, dimethyl sulfoxide.

DFOM partially rescued erastin-induced cell death, while Z-VAD-FMK and olaparib had no significant influence on it.

GPX4 expression is increased in the radioresistant cells and erastin inhibits GPX4 expression in the radioresistant cells. According to Yang et al (24), one of the mechanisms for erastin to induce cell death is that erastin inactivates GPX 
A

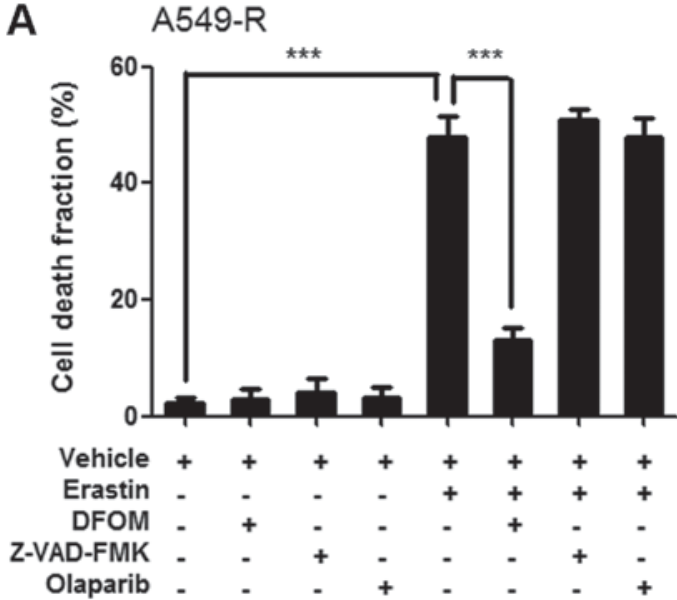

B

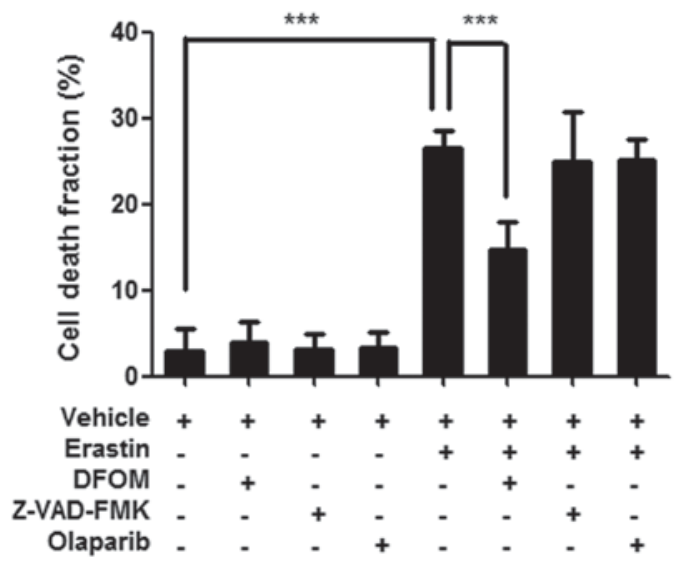

Figure 4. Treatment with erastin induces notable cell death. Treating cells with DFOM, Z-VAD-FMK and olaparib alone exhibited no significant effect on cell death. When cells were treated with DFOM and erastin together, cell death was decreased, compared with treatment with erastin alone. However, when cells were treated with Z-VAD-FMK and erastin, cell death exhibited no significance difference to the group treated with erastin alone. Additionally, olaparib had no significant effect on erastin-induced cell death. (A) Cell death fraction in A549-R cells. (B) Cell death fraction in H460-R cells. Data are presented as the mean \pm standard deviation. ${ }^{* * *} \mathrm{P}<0.001$. $\mathrm{R}$, radioresistant; DFOM, deferoxamine.

enzymes to trigger ferroptosis. GPX4 expression was detected in the radioresistant cells and GPX4 expression level was increased in A549-R and H460-R cells, compared with A549 and H460 cells, respectively (Fig. 5A). The radioresistant cells were treated with erastin and the expression of GPX4 was detected. The results demonstrated that GPX4 expression was suppressed by erastin in the A549-R and H460-R cells, compared with the control groups (Fig. 5B). Therefore, it was inferred that erastin increases radiosensitivity in NSCLC cells through inhibiting GPX4.

Knocking down GPX4 expression radiosensitizes NSCLCs cell to radiation in the radioresistant cell lines. It was inferred that high levels of GPX4 may contribute to radioresistance in NSCLC cells. Therefore, siRNA was used to knockdown GPX4 expression in A549-R and H460-R cells. The radiosensitivity of the GPX4-knockdown cells (siGPX4) and control cells (siMock) was subsequently detected with a colony formation

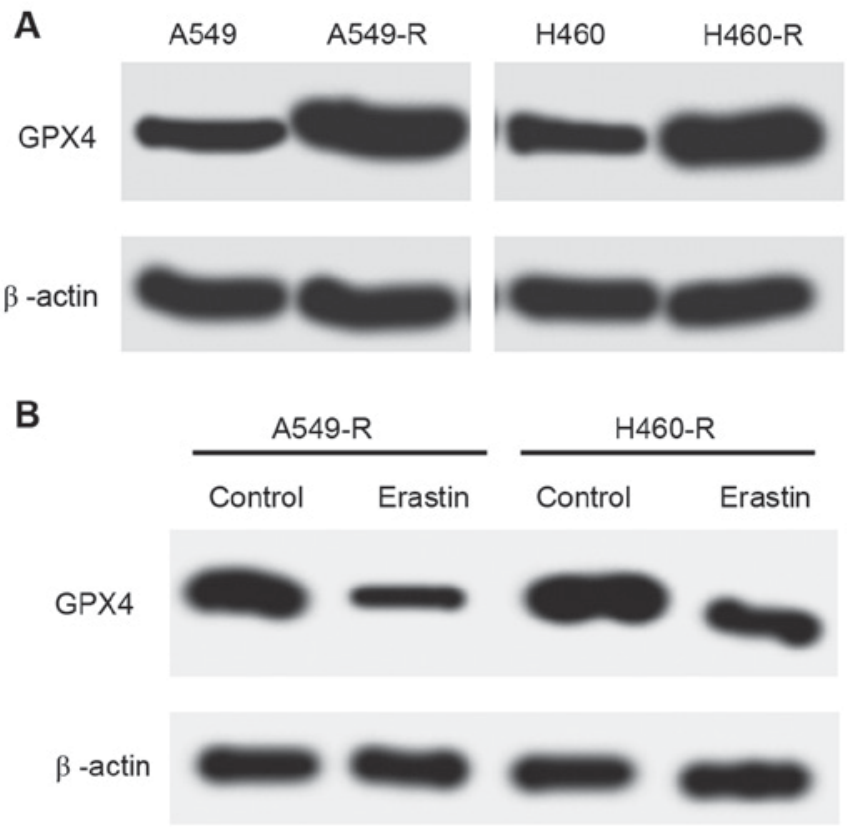

Figure 5. GPX4 expression detection in the radioresistant cells. GPX4 protein expression level was increased in A549-R and H460-R cells. Following treatment with erastin, GPX4 expression was markedly decreased. (A) GPX4 expression in the parental and radioresistant cells. (B) GPX4 expression in the control group and erastin group cells. R, radioresistant; GPX4, glutathione peroxidase 4 .

assay and the survival curves were calculated. In A549-R cells, siGPX4 cells exhibited decreased radioresistance, compared with siMock cells. In H460-R cells, similar results were achieved, demonstrating that knocking down the GPX4 expression resulted in decreased radioresistance (Fig. 6). The radiosensitivity parameters $\mathrm{D}_{0}, \mathrm{D}_{\mathrm{q}}, \mathrm{N}$ and $\mathrm{SF}_{2}$ are presented in Table III. Based on the results, it was concluded that knocking down the GPX4 expression resulted in decreased radioresistance.

Cell death induced by the knockdown of GPX4 could be partially rescued by DFOM, yet not rescued by Z-VAD-FMK and olaparib. Inhibiting GPX4 expression in A549-R and H460-R cells induced notable cell death. In A549-R cells, the cell death fraction was $56.03 \pm 3.63 \%$ in the siGPX4 group. In $\mathrm{H} 460-\mathrm{R}$ cells, the cell death fraction was $64.70 \pm 1.80 \%$ in the siGPX4 group. Treating siGPX4 group cells with DFOM decreased cell death fraction to $17.5 \pm 3.78 \%$ in A549-R cells. In H460-R cells, the cell death fraction was decreased to $16.00 \pm 3.05 \%$ following treatment with DFOM. However, treating cells with Z-VAD-FMK and olaparib did not significantly alter the cell death fraction (Fig. 7). Cell death fractions refer to the DMSO control. Collectively, these results indicated that the knockdown GPX4 decreases radioresistance in NSCLC cells by inducing ferroptosis.

\section{Discussion}

Erastin is a type of RSL, which can activate a lethal pathway that differs from other forms of cell death, such as apoptosis $(6,7)$. Erastin-induced cell death exhibits unique morphological, biochemical and genetic features, and the term ferroptosis was 
A
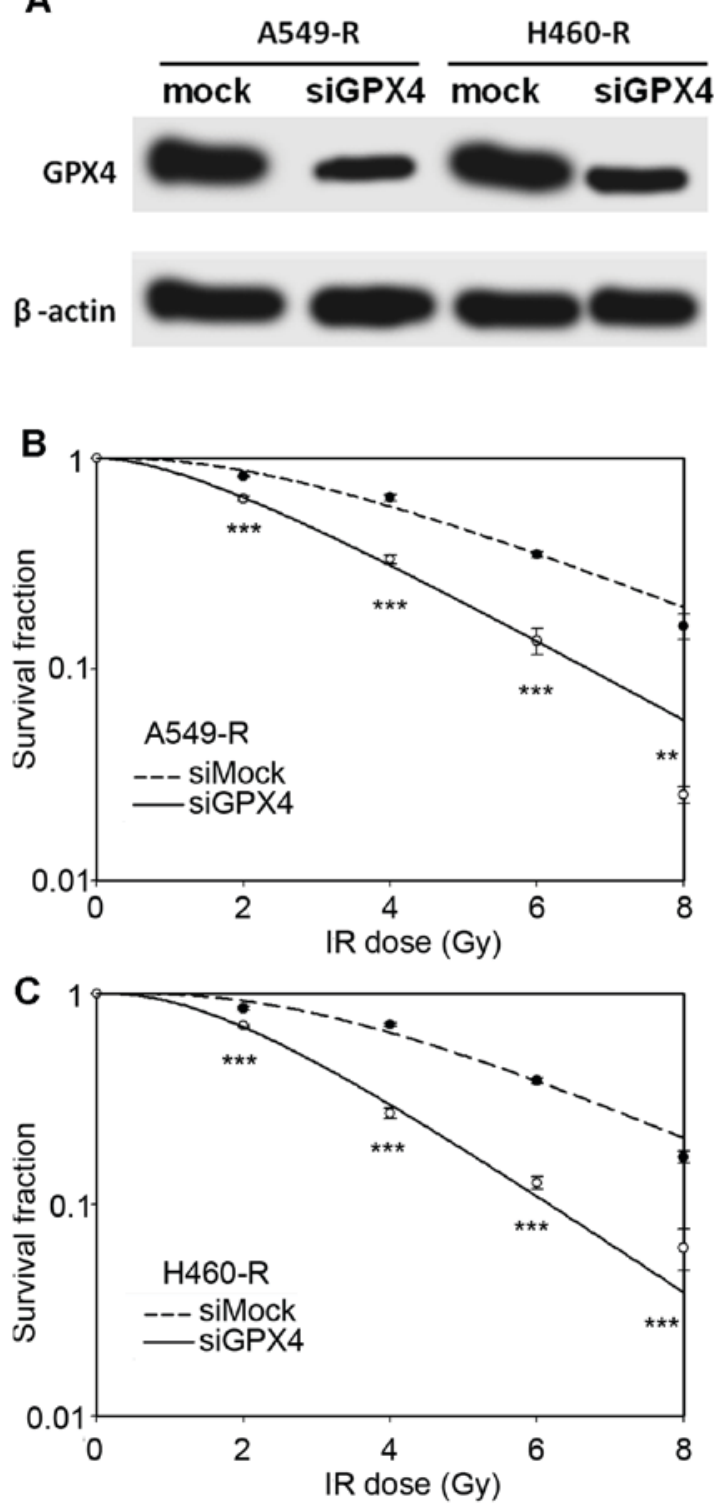

Figure 6. siRNA was used to knockdown GPX4 expression in radioresistant cells, and the radiosensitivity of the GPX4-knockdown cells (siGPX4) and control cells (siMock) was determined with a colony formation assay. siGPX4 cells exhibited decreased radioresistance, compared with siMock cells. (A) GPX4 expression in A549-R and H460-R cells. (B) Radiosensitivity of A549-R cells. (C) Radiosensitivity of H460-R cells. Data are presented as the mean \pm standard deviation. ${ }^{* *} \mathrm{P}<0.01$ and ${ }^{* * *} \mathrm{P}<0.001$. siRNA, small interfering RNA; GPX4, glutathione peroxidase 4; R, radioresistant; IR, irradiation.

proposed to describe this type of cell death (8). Iron chelators, including DFOM, can decrease the iron uptake of cells and inhibit ferroptosis $(9,10,27)$. However, apoptotic inhibitors, including Z-VAD-FMK, a caspase inhibitor, and olaparib, a PARP inhibitor, have no effect on ferroptosis $(12,28)$.

Ferroptosis is a form of cell death that is morphologically, biochemically and genetically different from apoptosis, necrosis and autophagy. Ferroptosis has previously been observed in RSL-treated cells (8-10). The classic characteristics of apoptosis include mitochondrial cytochrome $c$ release, caspase activation and chromatin fragmentation, which are absent in RSL-induced cell death $(7,8)$. However, increased levels of intracellular reactive oxygen species is commonly observed in RSL-treated cells $(9,10)$. Additionally,
Table III. Parameters of radiosensitivity in different groups.

\begin{tabular}{lcclll}
\hline & \multicolumn{2}{c}{ A549-R } & & \multicolumn{2}{c}{ H460-R } \\
\cline { 2 - 3 } \cline { 6 - 6 } Parameter & siMock & siGPX4 & & siMock & siGPX4 \\
\hline $\mathrm{D}_{0}$ & 3.11 & 2.27 & & 2.83 & 1.87 \\
$\mathrm{Dq}$ & 2.15 & 1.50 & & 2.37 & 1.66 \\
$\mathrm{~N}$ & 2.77 & 1.97 & & 3.78 & 2.83 \\
$\mathrm{SF}_{2}$ & 0.87 & 0.65 & & 0.92 & 0.69 \\
\hline
\end{tabular}

$\mathrm{D}_{0}$, mean lethal dose; $\mathrm{D}_{\mathrm{q}}$, quasi-threshold dose; $\mathrm{N}$, extrapolation number; $\mathrm{SF}_{2}$, surviving fraction at $2 \mathrm{~Gy}$; si, small interfering; $\mathrm{R}$, radioresistant; GPX4, glutathione peroxidase 4 .
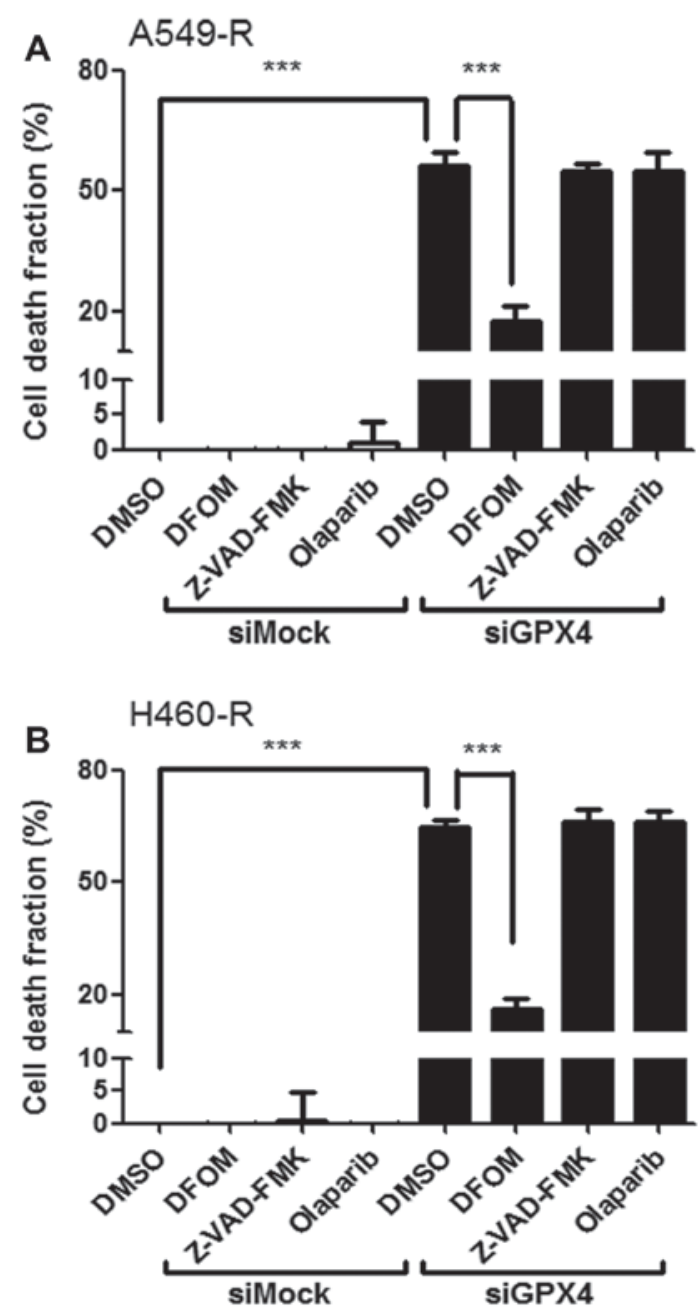

Figure 7. siRNA inhibition of GPX4 expression and cell death examination. Inhibiting GPX4 expression induced significant cell death. Treating siGPX4 group cells with DFOM significantly decreased cell death. However, treating cells with Z-VAD-FMK and olaparib did not significantly change the cell death fraction. (A) Cell death fraction in A549-R cells. (B) Cell death fraction in $\mathrm{H} 460-\mathrm{R}$ cells. Data are presented as the mean \pm standard deviation. ${ }^{* * * * *} \mathrm{P}<0.001$. siRNA, small interfering RNA; GPX4, glutathione peroxidase 4; $\mathrm{R}$, radioresistant; DFOM, deferoxamine; DMSO, dimethyl sulfoxide.

RSL-induced cell death can be prevented by iron chelation or the suppression of iron uptake $(7,8,24,29)$. Ferroptosis can be activated by small activators in Ras-expressing cancer 
cells (6,7,24). Therefore, the Ras-expressing A549 and H460 cells were selected for use in the present study. Firstly, radioresistant NSCLC cells were established (A549-R and H460-R). Subsequently, erastin was used to treat A549-R and H460-R cells. Treatment with erastin increased the sensitivity to radiation in A549-R and H460-R cells. Erastin and IR exhibited a combined effect on inducing cell death. DFOM partially rescued cell death induced by erastin while Z-VAD-FMK and olaparib had no notable effect on erastin-induced cell death. Collectively, the results indicated that erastin increased radiosensitivity in NSCLC by inducing ferroptosis, at least partially.

GPX4 expression has been demonstrated to be increased in radioresistant cells and GPX4 is a target molecular of erastin (24). In the present study, GPX4 expression was knocked-down in the radioresistant cells, and decreasing GPX4 expression was observed to significantly induce cell death. Cell death induced by GPX4-inhibition could be partially rescued by DFOM, and was not affected by treatment with Z-VAD-FMK and olaparib. The results indicated that knockdown of GPX4 induced ferroptosis. The knockdown of GPX4 in the radioresistance NSCLC cells resulted in increased cell death, which indicated that GPX4 may be involved in the regulation of radiosensitivity in NSCLC cells by modulating ferroptosis. Therefore, we hypothesized that erastin decreases radioresistance in NSCLC cells partially, by GPX4-mediated ferroptosis.

Ferroptosis has become the focus of an increasing number of studies. For example, in human hepatocellular carcinoma cells and other cancer cell lines, sorafenib has been demonstrated to induce ferroptosis $(30,31)$. Treatment with iron-containing water in tumor-bearing animals prior to undergoing radiotherapy also suppressed tumor growth by inducing ferroptosis $(14,15)$. Yu et al (12) also reported that ferroptosis induced enhanced sensitivity of acute myeloid leukemia cells to chemotherapy. In the present study, similar results were obtained by demonstrating that erastin radiosensitized NSCLC cells by inducing ferroptosis. These studies demonstrated that ferroptosis may participate in regulating sensitivity to chemotherapy and radiotherapy in different types of tumors.

The mechanism of ferroptosis has not been completely elucidated. However, GPX4 has demonstrated to be an essential in ferroptosis (24). One basic function of GPX4 is reducing the peroxides by consuming glutathione (GSH) (32). In 2008, Seiler et al (33) reported that a non-apoptotic form of cell death could be induced by deleting GPX4, and this form of cell death was mediated by lipid oxidation. In 2012, another study demonstrated that inhibiting the cystine/glutamate antiporter, system $\mathrm{XC}^{-}$, would elicit a novel form of cell death. System XC-is a transporter that provides cells with cysteine, which is an important material for GSH biosynthesis (8). GPX4 is the most important GSH-dependent enzyme in inducing this form of cell death (24). They named this novel form of cell death ferroptosis $(10,24)$. Although Kagan et al (34) demonstrated that one of the earliest events following GPX4 deletion is cardiolipin oxidation and phospholipid oxidation, the downstream mechanisms following GPX4 deletion remain largely unknown, and this mechanisms requires further study.
The present study, when taken in conjunction with the previous research, provides evidence of the therapeutic possibility of triggering ferroptosis in cancer cells. However, further research is required to understand more about ferroptosis, before it can be used as a cancer therapeutic in a clinical setting.

\section{Acknowledgements}

The authors would like to thank Professor Gang Wu and Professor Gang Peng for their advice and suggestions during the experiments.

\section{Funding}

The present study was supported by the Affiliated Hospital of Guangdong Medical College (grant no. 2017031795/1814).

\section{Availability of data and materials}

All data generated or analyzed during this study are included in this published article.

\section{Authors' contributions}

XP performed the colony formation assays and cell death assays. DJ established the radioresistant cell lines and performed the western blot analysis. YY and DY cultured the cells and participated in revising the manuscript. $\mathrm{HZ}$ and DZ analyzed the data. GP, ZC and ZY designed and instructed the study. SL, ZL and XP contributed to writing and revising the manuscript. GP gave final approval of the version to be published. All authors read and approved the final manuscript.

\section{Ethics approval and consent to participate}

Not applicable.

\section{Patient consent for publication}

Not applicable.

\section{Competing interests}

The authors declare that they have no competing interests.

\section{References}

1. Li K, Liu J, Tian M, Gao G, Qi X, Pan Y, Ruan J, Liu C and Su X: CHMP4C disruption sensitizes the human lung cancer cells to irradiation. Int J Mol Sci 17: pii: E18, 2015.

2. Noone AM, Howlader N, Krapcho M, Miller D, Brest A, Yu M, Ruhl J, Tatalovich Z, Mariotto A, Lewis DR, et al: SEER cancer statistics review (CSR), 1975-2015, National Cancer Institute. Bethesda, MD, https://seer.cancer.gov/csr/1975_2015/, based on November 2017 SEER data submission, posted to the SEER web site, April 2018.

3. Radovic M, Kanesvaran R, Rittmeyer A, Früh M, Minervini F, Glatzer M and Putora PM: Multidisciplinary treatment of lung cancer in older patients: A review. J Geriatr Oncol: Oct 3, 2018 (Epub ahead of print).

4. Gower A, Wang Y and Giaccone G: Oncogenic drivers, targeted therapies, and acquired resistance in non-small-cell lung cancer. J Mol Med (Berl) 92: 697-707, 2014. 
5. Nguyen KS, Neal JW and Wakelee H: Review of the current targeted therapies for non-small-cell lung cancer. World J Clin Oncol 5: 576-587, 2014.

6. Dolma S,Lessnick SL,Hahn WC and Stockwell BR: Identification of genotype-selective antitumor agents using synthetic lethal chemical screening in engineered human tumor cells. Cancer Cell 3: 285-296, 2003.

7. Yagoda N, von Rechenberg M, Zaganjor E, Bauer AJ, Yang WS Fridman DJ, Wolpaw AJ, Smukste I, Peltier JM, Boniface JJ, et al: RAS-RAF-MEK-dependent oxidative cell death involving voltage-dependent anion channels. Nature 447: 864-868, 2007.

8. Yang WS and Stockwell BR: Synthetic lethal screening identifies compounds activating iron-dependent, nonapoptotic cell death in oncogenic-RAS-harboring cancer cells. Chem Biol 15: 234-245, 2008 .

9. Dixon SJ and Stockwell BR: The role of iron and reactive oxygen species in cell death. Nat Chem Biol 10: 9-17, 2014.

10. Dixon SJ, Lemberg KM, Lamprecht MR, Skouta R, Zaitsev EM, Gleason CE, Patel DN, Bauer AJ, Cantley AM, Yang WS, et al: Ferroptosis: An iron-dependent form of nonapoptotic cell death. Cell 149: 1060-1072, 2012.

11. Wolpaw AJ, Shimada K, Skouta R, Welsch ME, Akavia UD, Pe'er D, Shaik F, Bulinski JC and Stockwell BR: Modulatory profiling identifies mechanisms of small molecule-induced cell death. Proc Natl Acad Sci USA 108: E771-E780, 2011

12. Yu Y, Xie Y, Cao L, Yang L, Yang M, Lotze MT, Zeh HJ, Kang R and Tang D: The ferroptosis inducer erastin enhances sensitivity of acute myeloid leukemia cells to chemotherapeutic agents. Mol Cell Oncol 2: e1054549, 2015.

13. Sun X, Niu X, Chen R, He W, Chen D, Kang R and Tang D Metallothionein-1G facilitates sorafenib resistance through inhibition of ferroptosis. Hepatology 64: 488-500, 2016.

14. Ivanov SD, Semenov AL, Mikhelson VM, Kovan'ko EG and Iamshanov VA: Effects of iron ion additional introduction in radiation therapy of tumor-bearing animals. Radiats Biol Radioecol 53: 296-303, 2013.

15. Ivanov SD, Semenov AL, Kovan'ko EG and Yamshanov VA: Effects of iron ions and iron chelation on the efficiency of experimental radiotherapy of animals with gliomas. Bull Exp Biol Med 158: 800-803, 2015.

16. El Osta BE, Behera M, Kim S, Berry LD, Sica G, Pillai RN, Owonikoko TK, Kris MG, Johnson BE, Kwiatkowski DJ, et al: Characteristics and outcomes of patients (pts) with metastatic KRAS mutant lung adenocarcinomas: Lung Cancer Mutation Consortium (LCMC) database. J Clin Oncol 35: (15 Suppl): S9021, 2017.

17. Kwon MY, Park E, Lee SJ and Chung SW: Heme oxygenase-1 accelerates erastin-induced ferroptotic cell death. Oncotarget 6 : 24393-24403, 2015

18. Yu JA, Li H, Meng X, Fullerton DA, Nemenoff RA, Mitchell JD and Weyant MJ: Group IIa secretory phospholipase expression correlates with group IIa secretory phospholipase inhibition-mediated cell death in K-ras mutant lung cancer cells J Thorac Cardiovasc Surg 144: 1479-1485, 2012.

19. Vigil D, Cherfils J, Rossman KL and Der CJ: Ras superfamily GEFs and GAPs: Validated and tractable targets for cance therapy? Nat Rev Cancer 10: 842-857, 2010.

20. Pearce AG, Segura TM, Rintala AC, Rintala-Maki ND and Lee $\mathrm{H}$ : The generation and characterization of a radiation-resistant model system to study radioresistance in human breast cancer cells. Radiat Res 156: 739-750, 2001.
21. Peng G, Cao RB, Li YH, Zou ZW, Huang J and Ding Q Alterations of cell cycle control proteins SHP1/2, p16, CDK4 and cyclin D1 in radioresistant nasopharyngeal carcinoma cells. Mol Med Rep 10: 1709-1716, 2014.

22. Hall EJ and Giaccia AJ.: Radiobiology for the radiologist. Section I, Chapter 3: Cell survival curves. 7th edition Wolters Kluwer Health/Lippincott Williams \& Wilkins, Philadelphia, PA, USA pp 32-51, 2012.

23. Wu L, Liu YY, Li ZX, Zhao Q, Wang X, Yu Y, Wang YY, Wang YQ and Luo F: Anti-tumor effects of penfluridol through dysregulation of cholesterol homeostasis. Asian Pac J Cancer Prev 15: 489-494, 2014

24. Yang WS, SriRamaratnam R, Welsch ME, Shimada K, Skouta R, Viswanathan VS, Cheah JH, Clemons PA, Shamji AF, Clish CB, et al: Regulation of ferroptotic cancer cell death by GPX4. Cell 156: 317-331, 2014

25. Tor YS, Yazan LS, Foo JB, Wibowo A, Ismail N, Cheah YK, Abdullah R, Ismail M, Ismail IS and Yeap SK: Induction of apoptosis in MCF-7 cells via oxidative stress generation, mitochondria-dependent and caspase-independent pathway by ethyl acetate extract of dillenia suffruticosa and its chemical profile. PLoS One 10: e0127441, 2015.

26. Yang KS, Kohler RH, Landon M, Giedt R and Weissleder R: Single cell resolution in vivo imaging of DNA damage following PARP inhibition. Sci Rep 5: 10129, 2015.

27. Li Y, Pan K, Chen L, Ning JL, Li X, Yang T, Terrando N, Gu J and Tao G: Deferoxamine regulates neuroinflammation and iron homeostasis in a mouse model of postoperative cognitive dysfunction. J Neuroinflammation 13: 268, 2016.

28. Fang S, Yu X, Ding H, Han J and Feng J: Effects of intracellular iron overload on cell death and identification of potent cell death inhibitors. Biochem Biophys Res Commun 503: 297-3029, 2018.

29. Jiang L, Kon N, Li T, Wang SJ, Su T, Hibshoosh H, Baer R and $\mathrm{Gu}$ W: Ferroptosis as a p53-mediated activity during tumour suppression. Nature 520: 57-62, 2015

30. Louandre C, Marcq I, Bouhlal H, Lachaier E, Godin C, Saidak Z, François C, Chatelain D, Debuysscher V, Barbare JC, et al: The retinoblastoma $(\mathrm{Rb})$ protein regulates ferroptosis induced by sorafenib in human hepatocellular carcinoma cells. Cancer Lett 356: 971-977, 2015

31. Lachaier E, Louandre C, Godin C, Saidak Z, Baert M, Diouf M, Chauffert B and Galmiche A: Sorafenib induces ferroptosis in human cancer cell lines originating from different solid tumors. Anticancer Res 34: 6417-6422, 2014

32. Conrad M and Friedmann Angeli JP: Glutathione peroxidase 4 (Gpx4) and ferroptosis: What's so special about it? Mol Cell Oncol 2: e995047, 2015.

33. Seiler A, Schneider M, Förster H, Roth S, Wirth EK, Culmsee C, Plesnila N, Kremmer E, Rådmark O, Wurst W, et al: Glutathione peroxidase 4 senses and translates oxidative stress into 12/15-lipoxygenase dependent- and AIF-mediated cell death. Cell Metab 8: 237-248, 2008

34. Kagan VE, Tyurin VA, Jiang J, Tyurina YY, Ritov VB, Amoscato AA, Osipov AN, Belikova NA, Kapralov AA, Kini V, et al: Cytochrome c acts as a cardiolipin oxygenase required for release of proapoptotic factors. Nat Chem Biol 1: 223-232, 2005 